\title{
Formation of Hubbard-like bands as a fingerprint of strong electron-electron interactions in FeSe
}

\author{
Matthew D. Watson, ${ }^{1,}{ }^{*}$ Steffen Backes, ${ }^{2}$ Amir A. Haghighirad, ${ }^{3}$ Moritz Hoesch, ${ }^{1}$ Timur K. Kim, ${ }^{1}$ \\ Amalia I. Coldea, ${ }^{3}$ and Roser Valenti ${ }^{2}$ \\ ${ }^{1}$ Diamond Light Source, Harwell Campus, Didcot OX11 ODE, United Kingdom \\ ${ }^{2}$ Institut für Theoretische Physik, Goethe-Universität Frankfurt, Max-von-Laue-Strasse 1, D-60438 Frankfurt am Main, Germany \\ ${ }^{3}$ Clarendon Laboratory, Department of Physics, University of Oxford, Parks Road, Oxford OX1 3PU, United Kingdom
}

(Received 8 December 2016; published 22 February 2017)

\begin{abstract}
We use angle-resolved photoemission spectroscopy (ARPES) to explore the electronic structure of single crystals of FeSe over a wide range of binding energies and study the effects of strong electron-electron correlations. We provide evidence for the existence of "Hubbard-like bands" at high binding energies consisting of incoherent many-body excitations originating from $\mathrm{Fe} 3 d$ states in addition to the renormalized quasiparticle bands near the Fermi level. Many high-energy features of the observed ARPES data can be accounted for when incorporating the effects of strong local Coulomb interactions in calculations of the spectral function via dynamical mean-field theory, including the formation of a Hubbard-like band. This shows that over the energy scale of several $\mathrm{eV}$, local correlations arising from the on-site Coulomb repulsion and Hund's coupling are essential for a proper understanding of the electronic structure of FeSe and other related iron-based superconductors.
\end{abstract}

DOI: 10.1103/PhysRevB.95.081106

Introduction. Understanding the role of electron-electron correlations in materials exhibiting high- $T_{c}$ unconventional superconductivity is one of the central problems within the field of strongly correlated electron systems. Unlike the cuprates, the parent compounds of the Fe-based superconductors (e.g., $\mathrm{LaFeAsO}$ ) are not Mott insulators but antiferromagnetic metals at low temperatures, away from half filling. Nevertheless, local electron-electron interactions on the $\mathrm{Fe}$ site do play an important role, although in this case it has been shown that it is the Hund's coupling $J_{H}$ rather than the Coulomb repulsion $U$ which is most important both for the magnetic ordering [1] and for the degree of band renormalization [2-11]. From an experimental point of view, clear manifestations of the effect of strong correlations in Fe-based superconductors are found in enhancements of quasiparticle effective masses deduced from specific heat [12] and quantum oscillation measurements [13], and from band renormalizations observed in angle-resolved photoemission spectroscopy (ARPES) [14-16]. These measurements indicate that the low-energy electronic structure broadly resembles that predicted by density functional theory (DFT) calculations, at least at temperatures above any magnetic or orbital orderings, but with the experimental band dispersions being renormalized by a factor typically of $\sim 3[14,15]$, although this varies substantially between systems, and is orbital dependent [5]. However, while general considerations of many-body theory would suggest that this band renormalization must be accompanied by the transfer of spectral weight into incoherent excitations at higher binding energies [17], the high-energy spectral weight has only rarely been experimentally investigated in $\mathrm{Fe}$-based superconductors $[15,18,19]$.

*Corresponding author: matthew.watson@diamond.ac.uk

Published by the American Physical Society under the terms of the Creative Commons Attribution 4.0 International license. Further distribution of this work must maintain attribution to the author(s) and the published article's title, journal citation, and DOI.
FeSe provides an ideal case to study the effect of strong correlations in Fe-based superconductors. The recent availability of high-quality single crystals [20,21] and thin films [22] of FeSe has led to a surge of experimental work, including recent ARPES studies with a focus on the origin of the nematic phase [21,23-26]. ARPES [16,21], quantum oscillations [21,27,28], and specific heat measurements of FeSe [20] have previously reported significant orbital-dependent effective mass renormalizations. Theoretically, a significant effect of correlations in FeSe has been found in combined density functional theory with dynamical mean-field theory (DFT+DMFT) calculations, where mass enhancement factors of $\sim 2.3$ have been reported when accounting for a local static Coulomb density-density interaction on the Fe site [29], or even higher values of 3-5 when including spin-flip and pair-hopping terms $[5,30]$.

In this Rapid Communication, we present systematic ARPES studies of the spectral function of FeSe to high binding energies. In addition to the renormalized quasiparticle bands near the Fermi level, we find much broader features lying in a range of 1-2.5 eV binding energy, well separated from the quasiparticle structure and the Se $4 p$ bands at $\sim 3-6 \mathrm{eV}$. A "peak-dip-hump" structure on such an energy scale is usually a trademark of strong electron-electron interactions, which reduce the spectral weight of the quasiparticle peak and give rise to Hubbard bands at higher and lower binding energies [31]. Our DFT+DMFT calculations are able to reproduce many of the qualitative features of the experimental electronic structure at high binding energies, including the formation of Hubbard-like bands of incoherent spectral weight. While accounting for local electron-electron interactions within DFT+DMFT alone is not sufficient for a perfect description of the experimental Fermi surface, we show that the strong interactions are responsible for the overall form of the spectral function of FeSe over an energy scale of several $\mathrm{eV}$.

Methods. Single crystals of FeSe were grown by the vapor-transport method [21]. ARPES measurements were performed at the I05 beamline at Diamond Light Source at temperatures below $10 \mathrm{~K}$. ARPES measurements are a probe 
of the one-particle spectral function $A(\omega, \mathbf{k})$ [17], multiplied by the Fermi occupation function and the matrix elements for photoemission [17], with some additional background. This spectral function is commonly expressed as

$$
A(\omega, \mathbf{k})=-\frac{1}{\pi} \frac{\Sigma^{\prime \prime}(\omega, \mathbf{k})}{\left[\omega+\mu-\epsilon_{\mathbf{k}}^{b}-\Sigma^{\prime}(\omega, \mathbf{k})\right]^{2}+\left[\Sigma^{\prime \prime}(\omega, \mathbf{k})\right]^{2}},
$$

where $\epsilon_{\mathbf{k}}^{b}$ is the bare noninteracting dispersion, $\mu$ the chemical potential, and $\Sigma^{\prime}$ and $\Sigma^{\prime \prime}$ are the real and imaginary parts of the self-energy, which in general is orbital, frequency, and momentum dependent. In many materials where electronic correlations are weak and do not play a significant role, $\Sigma$ is small and sharp dispersions can be observed in ARPES measurements to binding energies of several $\mathrm{eV}$, usually in good agreement with the DFT dispersions. On the other hand, in $\mathrm{FeSe}$, electron-electron interactions on the $\mathrm{Fe} 3 d$ site do give a significant contribution to the self-energy $[29,30]$, while the system remains metallic. Therefore, the observed dispersions close to the Fermi level at low temperatures can be interpreted as coherent quasiparticles with renormalized dispersions $\epsilon_{\mathrm{k}}^{q}=$ $\epsilon_{\mathbf{k}}^{b}+\Sigma^{\prime}$, and a scattering rate $\Sigma^{\prime \prime}$ that introduces a finite lifetime for quasiparticle excitations. Depending on the form of $\Sigma(\omega, \mathbf{k})$ there may be apparent "kinks" or "waterfalls" [32] in the spectral function where the observed states transform from the renormalized quasiparticle peak close to the Fermi level into incoherent excitations at higher or lower binding energies. Generally speaking, at higher binding energy, features can become very broad and incoherent when $\Sigma^{\prime \prime}$ becomes large, and in particular the formation of Hubbard-like bands is possible $[33,34]$. While experimental evidence of Hubbard bands has been largely reported for effective one-band systems [17,35], results for multiorbital systems are scarce, with only a few well-studied exceptions such as transition metal oxides [36-39].

The DFT+DMFT calculations were performed full charge self-consistently within the local density approximation in DFT and using the full-potential linear augmented planewave (FLAPW) basis (without spin-orbit coupling) within the WIEN2K [40] package. Calculations were done for the orthorhombic crystal structure [41], and differences in the calculation to the tetragonal crystal structures were small [see the Supplemental Material (SM) [42]. We used the projection method onto a local basis as described in Refs. [3,43], with a window encompassing both the iron $3 d$ and selenium $4 p$ states. The impurity problem for the $\mathrm{Fe} 3 d$ orbitals was solved with the strong-coupling continuous-time quantum Monte Carlo method [44] using the ALPS package [45]. As interaction parameters we use the established values of $U=$ $4 \mathrm{eV}, J_{H}=0.8 \mathrm{eV}[30,46]$. We employed the fully localized limit $[47,48]$ for the double counting term, and the stochastic analytic continuation method for obtaining real-frequency data [49]. Calculations were performed at a temperature of $\beta=100 \mathrm{eV}^{-1}$, corresponding to $T=116 \mathrm{~K}$.

Results. In Fig. 1 we present high-symmetry ARPES measurements for $\mathrm{FeSe}$ in the $M-\Gamma-M$ direction, using linear vertical (LV) polarization. In this geometry, strong matrix element effects dictate that the spectral weight arises overwhelmingly from a single holelike band with $d_{y z}$ character [21], which simplifies the observation. Figure 1(a) focuses
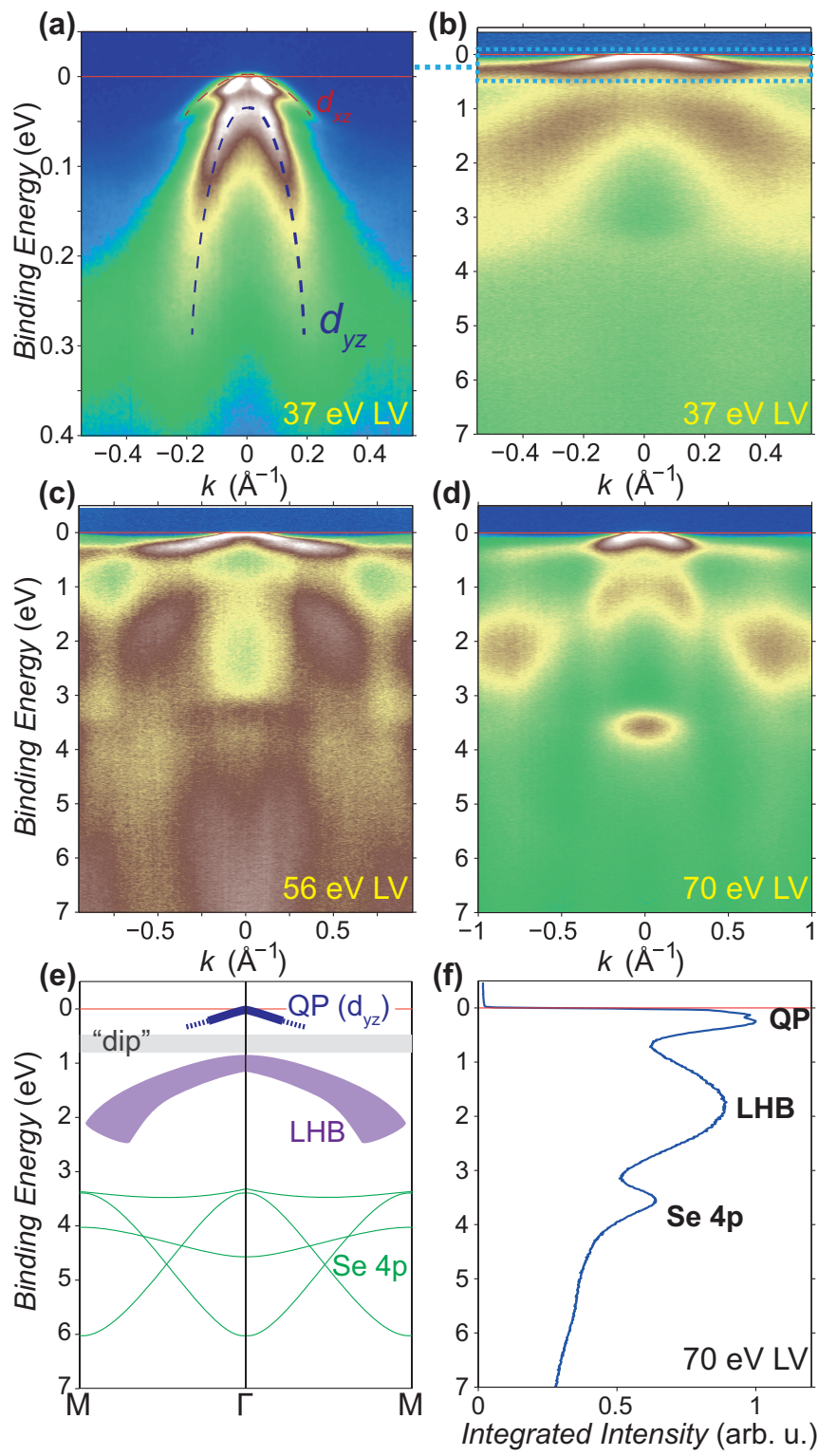

FIG. 1. (a)-(d) ARPES data in the $M-\Gamma-M$ direction at $37 \mathrm{eV}$ in linear vertical (LV) polarization at $10 \mathrm{~K}$. In this geometry a holelike quasiparticle band with $d_{y z}$ orbital character dominates the photoemission spectrum. (b)-(d) Measurements in the same geometry at different incident photon energies. The data extend to high binding energies, where much broader features are found. (e) Schematic of the high-energy spectrum. (f) Integrated spectral weight from (d), showing features associated with the quasiparticle (QP), lower Hubbard-band (LHB) intensities, as well as a contribution from the Se $4 p$ bands.

on the dispersion of this $d_{y z}$ hole band close to $E_{F}$. The quasiparticle band dispersions undergo $\sim 20 \mathrm{meV}$ band shifts in the nematic phase [26], but these are very small perturbations on the energy scales of a few $\mathrm{eV}$ as considered in this Rapid Communication. Due to spin-orbit coupling there is a small mixing of spectral weight onto the outer $\left(d_{x z}\right)$ hole band near the Fermi level [21]. In Figs. 1(b)-1(d) we present measurements extending to binding energies of $7 \mathrm{eV}$ at a selection of incident photon energies. Varying the photon 

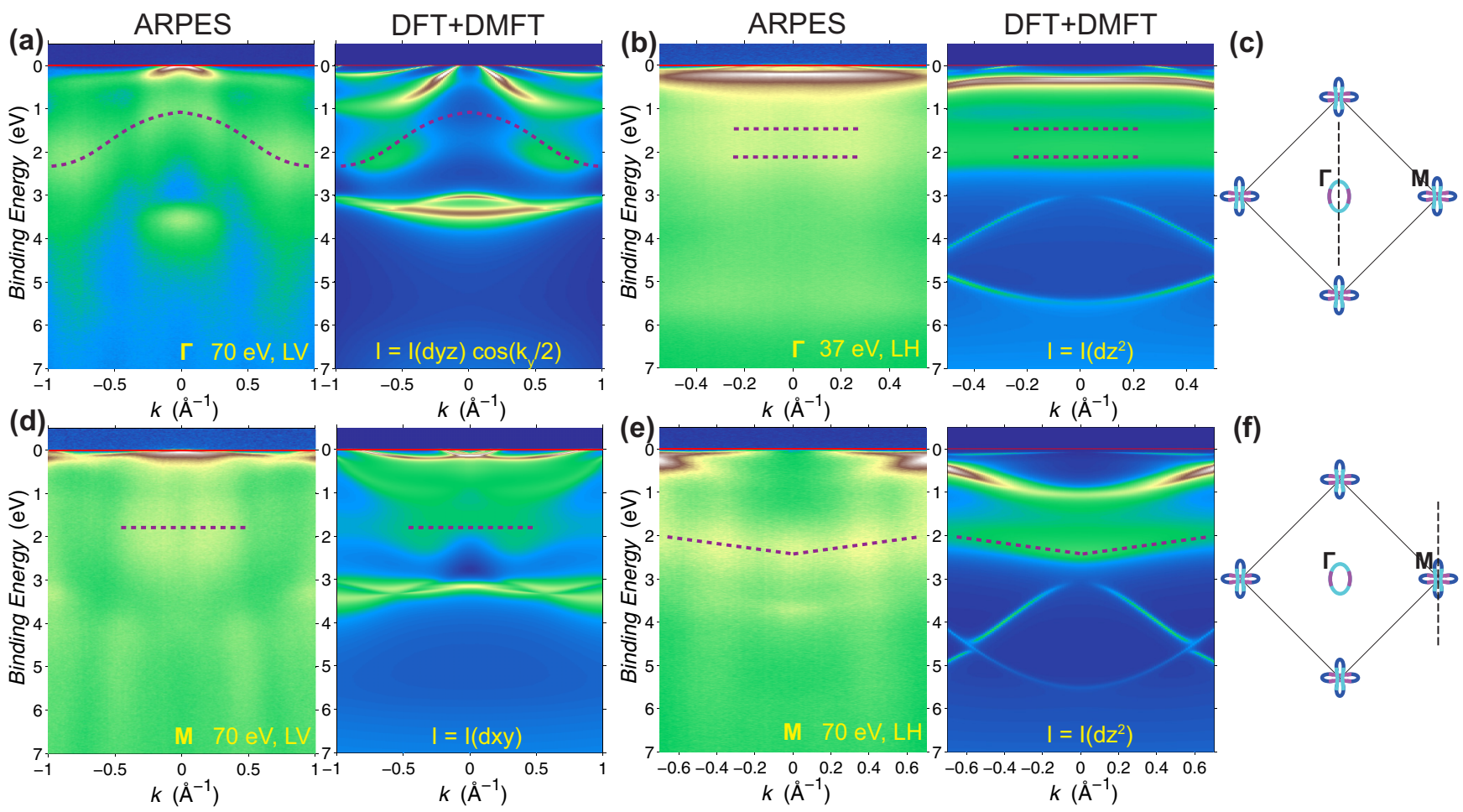

FIG. 2. (a), (b), (d), (e) Comparison of ARPES spectra with DFT+DMFT calculations. The DFT+DMFT simulations are obtained by applying simple selection rules to the orbitally resolved spectral weight, to account for the experimental matrix element effects. Dashed lines are guides to the eye showing the location of experimental incoherent Fe $3 d$ spectral weight. (c), (f) Schematic measurement geometries of the cuts shown in (a) and (b) (and also Fig. 1) and (d) and (e), respectively.

energy has multiple effects. First, the $k_{z}$ of the slice of the Brillouin zone probed varies (e.g., 37 and $56 \mathrm{eV}$ are near the $\Gamma$ and $Z$ points, respectively [21]) which can affect the position and orbital character of bands. Second, ARPES matrix elements themselves have a complex photon-energy dependence. Finally, if the photon energy passes through an $\mathrm{Fe}$ or Se resonance, this may affect the relative intensity of $\mathrm{Fe}$ or Se contributions to the photoemission [this gives an enhancement of the Se bands in the $56 \mathrm{eV}$ spectra in Fig. 1(c)]. We do not attempt to disentangle all these effects which lead to the differences between spectra presented in Figs. 1(b)-1(d), but rather point out five common features which are observed at all photon energies, as we have represented schematically in Figs. 1(e) and 1(f): (i) Near the Fermi level the observed band is both shifted and renormalized with respect to DFT calculations, as has been widely reported in Fe-based superconductors [50,51], although the much smaller than expected Fermi surfaces in FeSe are a unique feature. (ii) The quasiparticle band dispersions become much sharper towards the Fermi level. (iii) There is generally a dip in intensity in the range $\sim 0.5-1 \mathrm{eV}$ in experiments, where neither quasiparticles nor incoherent excitations are found. (iv) Traces of the Se $4 p$ bands are detected in the range 3-6 eV binding energy, as predicted by DFT. Therefore, the Se $4 p$ bands do not experience any significant renormalization. Finally, (v) in the range of $\sim 1-2.5 \mathrm{eV}$ we observe an anomalous broadband of intensity which cannot be attributed to either a $\mathrm{Fe} 3 d$ quasiparticle band or a Se $4 p$ band. The width of this spectral feature is of the order of $\sim 1 \mathrm{eV}$, which indicates that these excitations are very short lived. We interpret this as a "Hubbard-like band," consisting of incoherent spectral weight that is a precursor of the localized electron-removal states, the lower Hubbard band, in Mott-Hubbard-insulating systems. No significant temperature dependence was found in the high-energy features up to $150 \mathrm{~K}$ (SM).

In Fig. 2 we present a selection of ARPES spectra obtained in different measurement conditions, which indicate that this incoherent spectral weight in the region around $\sim 1-2.5 \mathrm{eV}$ is a general feature of FeSe, and not specific to a particular band or geometry. Next to each experimental measurement, we also show how the high-energy features of FeSe seen by ARPES can be qualitatively reproduced by calculations of the spectral function in DFT+DMFT. In order to perform a comparison to ARPES data, simple selection rules are employed to simulate the photoemission matrix elements in that geometry. They are based on both symmetry considerations and the identified orbital character of the primary quasiparticle bands in the cut [26,52]. As presented in Fig. 2(a), DFT+DMFT reproduces the observed renormalized quasiparticle $d_{y z}$ band and some additional high-energy spectral weight around 1-2.5 eV. However, the agreement is not perfect, and the renormalization of the effective masses in DFT+DMFT (e.g., $m^{*} / m_{\mathrm{LDA}}=$ 2.09 for $d_{x z / y z}$, see the SM) is less than the experiments ( $\sim 2-4$ for $d_{x z / y z}$ bands [21]), which is to be expected due to the neglect of spin-flip and pair-hopping terms [30] and dynamical screening effects $[53,54]$. Still, we expect that any Hubbard-band-like features are not qualitatively affected by these approximations, since their binding energy is governed 

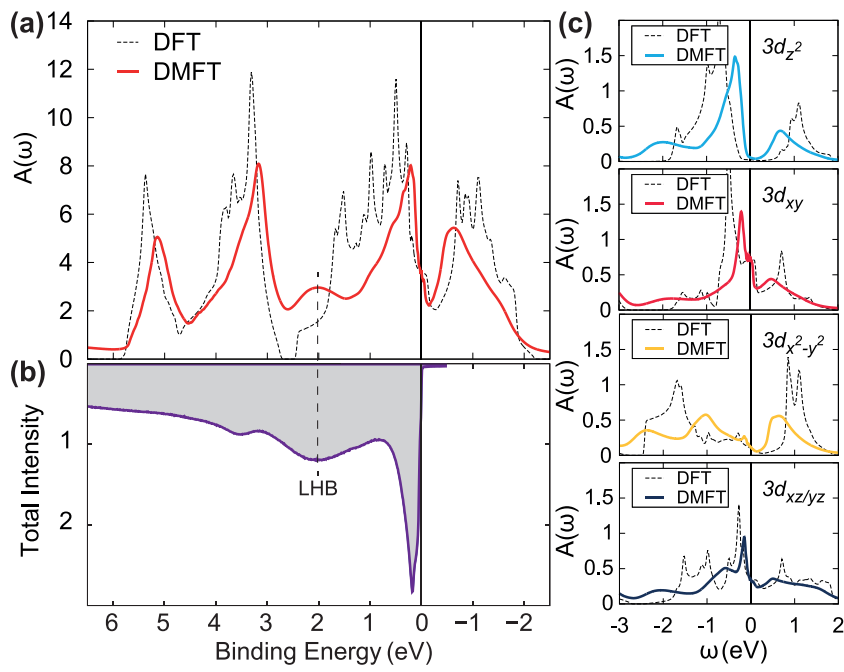

(d)
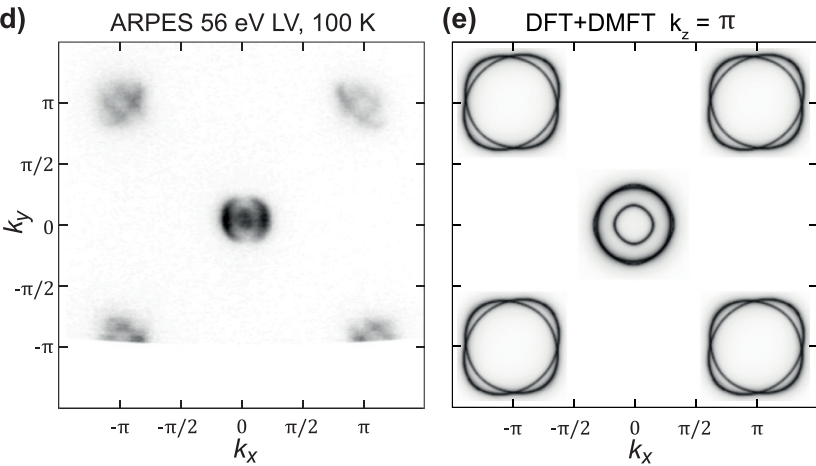

FIG. 3. (a) Integrated spectral weights as calculated from DFT and DFT+DMFT. (b) Total intensity obtained by summation over the different cuts in Figs. 2(a), 2(c), and 2(d). (c) Orbital-resolved spectral weight. (d) Comparison of Fermi surfaces determined experimentally at $100 \mathrm{~K}$ in the tetragonal phase and (e) as calculated by DFT+DMFT projected in the $k_{z}=\pi$ plane.

by the low-energy static values of the interaction, which are accounted for in the calculation. In Fig. 2(b), the DFT+DMFT calculation shows a broadband of incoherent $d_{z^{2}}$ spectral weight in good correspondence with the anomalous weight found in ARPES around 1.5-2.5 eV. In Fig. 2(d), DFT+DMFT finds some incoherent spectral weight in the $d_{x y}$ orbital around the $M$ point, similar to the ARPES data. Finally, in Fig. 2(e), the $d_{z^{2}}$ weight through the $M$ point is reproduced very well, showing a clear formation of a Hubbard-like band. Overall, there is a qualitative good agreement between calculations and experiment, as the DFT+DMFT technique correctly captures both the renormalized quasiparticle bands which sharpen approaching the Fermi level, along with the incoherent spectral weight around 1-2.5 eV.

In Fig. 3(a), we compare the integrated spectral weight from our DFT+DMFT calculation with the result from DFT. Notably, the Fe $3 d$ bandwidth develops a peak-dip-hump structure which is not present in the DFT; this arises from the separation of the quasiparticle bands and the Hubbard satellite peak around $2 \mathrm{eV}$. As expected, the DMFT treatment does not strongly affect the Se $4 p$ bands. In Fig. 3(c), we show the different orbital contributions to the total spectral weight.
The Hubbard-band feature appears most clearly in the $d_{z^{2}}$ and $d_{x z / y z}$ orbitals but can be identified in all, similar to Ref. [30]. In Fig. 3(b), we compare the total calculated spectral weight with a summation of the experimental data from Figs. 2(a), 2(c), and 2(d). Similar qualitative features are found, with good agreement on the position of the Hubbard-like peak, which supports the chosen values of the interaction parameters $U, J_{H}$, which are also close to values recently determined from firstprinciples calculations [55]. We checked that in DFT+DMFT the Hubbard-like peak indeed behaves as a Hubbard band in the sense that its spectral weight shifts to higher binding energies with increasing $U, J_{H}(\mathrm{SM})$. Especially, we find that the Hund's coupling $J_{H}$ has a stronger effect on the energy of the Hubbard band than $U$ (SM).

Finally, in Figs. 3(d) and 3(e), we compare the experimental Fermi surfaces of FeSe at $100 \mathrm{~K}$ with the calculated ones. The measured Fermi surfaces are significantly shrunk compared to the prediction of DFT+DMFT. In order to match the experimental dispersions, the real parts of the self-energies would need to be significantly momentum dependent in order to introduce a downward shift for hole bands at the Gamma point and an upward shift for the electron bands at the $M$ point [21,51]. In DMFT, the considered interactions $\left(U, J_{H}\right)$ are purely local and the self-energies $\Sigma(\omega)$ are independent of $\mathbf{k}$, albeit orbital dependent, so that momentum-dependent shifts of the DFT band structure can only result from the momentumdependent orbital characters of the bands. The limitations of DFT+DMFT at the Fermi level indicate that effects not included in the calculations such as nonlocal intersite interactions [56], coupling to bosonic modes [57]. or frustrated magnetism [58] are likely to be relevant to the low-energy physics. However, for the wide energy scales considered here, our DFT+DMFT calculation is able to satisfactorily capture many of the high-energy features of our ARPES spectra, including the presence of incoherent spectral weight in the form of Hubbard-like bands at high binding energies, with specific orbital-dependent agreements. Our experiments and calculations place bulk FeSe as a significantly correlated metal, with coherent quasiparticles at the Fermi level, but also exhibiting incoherent spectral weight at high binding energies, consistent with earlier photoemission studies [59].

Conclusion. To summarize, we have provided systematic experimental evidence, backed up by theoretical DFT+DMFT calculations, for the emergence of a Fe $3 d$ Hubbard-like band in the spectral function of FeSe, distinct from the quasiparticle states near the Fermi level. This high-energy feature is interpreted as a fingerprint of the effect of strong electron-electron correlations. Despite the strong renormalization and shift of spectral weight into the Hubbard-like features, a well-defined quasiparticle peak at the Fermi level is retained. Therefore, FeSe provides a rare opportunity to study Hubbard-band physics in a significantly correlated, metallic, multiorbital system. The unique properties of FeSe continue to provide theoretical challenges, but we have demonstrated that the DFT+DMFT technique captures the essential features of the high-energy spectral function well, highlighting the importance of local Coulomb interactions and Hund's coupling for both low- and high-energy features in Fe-based superconductors. 
Acknowledgmens. We thank S. Biermann, B. Büchner, D. Guterding, H. Iwasawa, H. O. Jeschke, and L. C. Rhodes for useful discussions. We acknowledge Diamond Light Source for time on beamline I05 under Proposals No. CM12153 and No. SI10203. S.B. and R.V. thank the Deutsche Forschungsgemeinschaft (DFG) for financial support through
Grant No. SPP 1458. Part of the work was supported by the EPSRC (EP/L001772/1, EP/I004475/1, EP/I017836/1). A.A.H. acknowledges the financial support of the Oxford Quantum Materials Platform Grant (No. EP/M020517/1). A.I.C. acknowledges an EPSRC Career Acceleration Fellowship (Grant No. EP/I004475/1).
[1] M. D. Johannes and I. I. Mazin, Microscopic origin of magnetism and magnetic interactions in ferropnictides, Phys. Rev. B 79, 220510(R) (2009).

[2] K. Haule and G. Kotliar, Coherence-incoherence crossover in the normal state of iron oxypnictides and importance of Hund's rule coupling, New J. Phys. 11, 025021 (2009).

[3] M. Aichhorn, L. Pourovskii, V. Vildosola, M. Ferrero, O. Parcollet, T. Miyake, A. Georges, and S. Biermann, Dynamical mean-field theory within an augmented plane-wave framework: Assessing electronic correlations in the iron pnictide $\mathrm{LaFeAsO}$, Phys. Rev. B 80, 085101 (2009).

[4] L. de' Medici, J. Mravlje, and A. Georges, Janus-Faced Influence of Hund's Rule Coupling in Strongly Correlated Materials, Phys. Rev. Lett. 107, 256401 (2011).

[5] Z. P. Yin, K. Haule, and G. Kotliar, Kinetic frustration and the nature of the magnetic and paramagnetic states in iron pnictides and iron chalcogenides, Nat. Mater. 10, 932 (2011).

[6] R. Yu and Q. Si, Mott transition in multiorbital models for iron pnictides, Phys. Rev. B 84, 235115 (2011).

[7] J. Ferber, K. Foyevtsova, R. Valentí, and H. O. Jeschke, LDA+DMFT study of the effects of correlation in LiFeAs, Phys. Rev. B 85, 094505 (2012).

[8] J. Ferber, H. O. Jeschke, and R. Valentí, Fermi Surface Topology of LaFePO and LiFeP, Phys. Rev. Lett. 109, 236403 (2012).

[9] A. Georges, L. de' Medici, and J. Mravlje, Strong correlations from Hund's coupling, Annu. Rev. Condens. Matter Phys. 4, 137 (2013).

[10] S. Backes, H. O. Jeschke, and R. Valentí, Microscopic nature of correlations in multiorbital $A \mathrm{Fe}_{2} \mathrm{As}_{2}(A=\mathrm{K}, \mathrm{Rb}, \mathrm{Cs})$ : Hund's coupling versus Coulomb repulsion, Phys. Rev. B 92, 195128 (2015).

[11] L. Fanfarillo and E. Bascones, Electronic correlations in Hund metals, Phys. Rev. B 92, 075136 (2015).

[12] F. Hardy, A. E. Böhmer, L. de' Medici, M. Capone, G. Giovannetti, R. Eder, L. Wang, M. He, T. Wolf, P. Schweiss, R. Heid, A. Herbig, P. Adelmann, R. A. Fisher, and C. Meingast, Strong correlations, strong coupling, and $s$-wave superconductivity in hole-doped $\mathrm{BaFe}_{2} \mathrm{As}_{2}$ single crystals, Phys. Rev. B 94, 205113 (2016).

[13] C. Putzke, A. I. Coldea, I. Guillamón, D. Vignolles, A. McCollam, D. LeBoeuf, M. D. Watson, I. I. Mazin, S. Kasahara, T. Terashima, T. Shibauchi, Y. Matsuda, and A. Carrington, de Haas-van Alphen Study of the Fermi Surfaces of Superconducting LiFeP and LiFeAs, Phys. Rev. Lett. 108, 047002 (2012).

[14] S. V. Borisenko, V. B. Zabolotnyy, D. V. Evtushinsky, T. K. Kim, I. V. Morozov, A. N. Yaresko, A. A. Kordyuk, G. Behr, A. Vasiliev, R. Follath, and B. Büchner, Superconductivity without Nesting in LiFeAs, Phys. Rev. Lett. 105, 067002 (2010).

[15] D. V. Evtushinsky, A. N. Yaresko, V. B. Zabolotnyy, J. Maletz, T. K. Kim, A. A. Kordyuk, M. S. Viazovska, M. Roslova,
I. Morozov, R. Beck, S. Wurmehl, H. Berger, B. Büchner, and S. V. Borisenko, Anomalous high-energy electronic interaction in iron-based superconductor, arXiv:1409.1537.

[16] J. Maletz, V. B. Zabolotnyy, D. V. Evtushinsky, S. Thirupathaiah, A. U. B. Wolter, L. Harnagea, A. N. Yaresko, A. N. Vasiliev, D. A. Chareev, A. E. Böhmer, F. Hardy, T. Wolf, C. Meingast, E. D. L. Rienks, B. Büchner, and S. V. Borisenko, Unusual band renormalization in the simplest iron-based superconductor FeSe, Phys. Rev. B 89, 220506 (2014).

[17] R. Comin and A. Damascelli, ARPES: A probe of electronic correlations, arXiv:1303.1438.

[18] D. V. Evtushinsky, M. Aichhorn, Y. Sassa, Z.-H. Liu, J. Maletz, T. Wolf, A. N. Yaresko, S. Biermann, S. V. Borisenko, and B. Buchner, Direct observation of dispersive lower Hubbard band in iron-based superconductor FeSe, arXiv:1612.02313.

[19] I. A. Nekrasov, N. S. Pavlov, and M. V. Sadovskii, Electronic structure of NaFeAs superconductor: LDA+DMFT calculations compared to the ARPES experiment, JETP Lett. 102, 26 (2015).

[20] A. E. Böhmer, T. Arai, F. Hardy, T. Hattori, T. Iye, T. Wolf, H. v. Löhneysen, K. Ishida, and C. Meingast, Origin of the Tetragonal-to-Orthorhombic Phase Transition in FeSe: A Combined Thermodynamic and NMR Study of Nematicity, Phys. Rev. Lett. 114, 027001 (2015).

[21] M. D. Watson, T. K. Kim, A. A. Haghighirad, N. R. Davies, A. McCollam, A. Narayanan, S. F. Blake, Y. L. Chen, S. Ghannadzadeh, A. J. Schofield, M. Hoesch, C. Meingast, T. Wolf, and A. I. Coldea, Emergence of the nematic electronic state in FeSe, Phys. Rev. B 91, 155106 (2015).

[22] S. Tan, Y. Zhang, M. Xia, Z. Ye, F. Chen, X. Xie, R. Peng, D. Xu, Q. Fan, H. Xu, J. Jiang, T. Zhang, X. Lai, T. Xiang, J. Hu, B. Xie, and D. Feng, Interface-induced superconductivity and strain-dependent spin density waves in $\mathrm{FeSe} / \mathrm{SrTiO}_{3}$ thin films. Nat. Mater. 12, 634 (2013).

[23] P. Zhang, T. Qian, P. Richard, X. P. Wang, H. Miao, B. Q. Lv, B. B. Fu, T. Wolf, C. Meingast, X. X. Wu, Z. Q. Wang, J. P. Hu, and $H$. Ding, Observation of two distinct $d_{x z} / d_{y z}$ band splittings in FeSe, Phys. Rev. B 91, 214503 (2015).

[24] Y. Suzuki, T. Shimojima, T. Sonobe, A. Nakamura, M. Sakano, H. Tsuji, J. Omachi, K. Yoshioka, M. Kuwata-Gonokami, T. Watashige, R. Kobayashi, S. Kasahara, T. Shibauchi, Y. Matsuda, Y. Yamakawa, H. Kontani, and K. Ishizaka, Momentum-dependent sign inversion of orbital polarization in superconducting FeSe, Phys. Rev. B 92, 205117 (2015).

[25] M. D. Watson, T. K. Kim, A. A. Haghighirad, S. F. Blake, N. R. Davies, M. Hoesch, T. Wolf, and A. I. Coldea, Suppression of orbital ordering by chemical pressure in $\mathrm{FeSe}_{1-x} \mathrm{~S}_{x}$, Phys. Rev. B 92, 121108 (2015).

[26] M. D. Watson, T. K. Kim, L. C. Rhodes, M. Eschrig, M. Hoesch, A. A. Haghighirad, and A. I. Coldea, Evidence for unidirectional nematic bond ordering in FeSe, Phys. Rev. B 94, 201107 (2016). 
[27] M. D. Watson, T. Yamashita, S. Kasahara, W. Knafo, M. Nardone, J. Béard, F. Hardy, A. McCollam, A. Narayanan, S. F. Blake, T. Wolf, A. A. Haghighirad, C. Meingast, A. J. Schofield, H. v. Löhneysen, Y. Matsuda, A. I. Coldea, and T. Shibauchi, Dichotomy between the Hole and Electron Behavior in Multiband Superconductor FeSe Probed by Ultrahigh Magnetic Fields, Phys. Rev. Lett. 115, 027006 (2015).

[28] T. Terashima, N. Kikugawa, A. Kiswandhi, E.-S. Choi, J. S. Brooks, S. Kasahara, T. Watashige, H. Ikeda, T. Shibauchi, Y. Matsuda, T. Wolf, A. E. Böhmer, F. Hardy, C. Meingast, H. Löhneysen, M.-T. Suzuki, R. Arita, and S. Uji, Anomalous Fermi surface in FeSe seen by Shubnikov-de Haas oscillation measurements, Phys. Rev. B 90, 144517 (2014).

[29] I. Leonov, S. L. Skornyakov, V. I. Anisimov, and D. Vollhardt, Correlation-Driven Topological Fermi Surface Transition in FeSe, Phys. Rev. Lett. 115, 106402 (2015).

[30] M. Aichhorn, S. Biermann, T. Miyake, A. Georges, and M. Imada, Theoretical evidence for strong correlations and incoherent metallic state in FeSe, Phys. Rev. B 82, 064504 (2010).

[31] X. Y. Zhang, M. J. Rozenberg, and G. Kotliar, Mott Transition in the $d=\infty$ Hubbard Model at Zero Temperature, Phys. Rev. Lett. 70, 1666 (1993).

[32] H. Iwasawa, Y. Yoshida, I. Hase, K. Shimada, H. Namatame, M. Taniguchi, and Y. Aiura, High-Energy Anomaly in the Band Dispersion of the Ruthenate Superconductor, Phys. Rev. Lett. 109, 066404 (2012).

[33] G. Kotliar and D. Vollhardt, Strongly correlated materials: Insights from dynamical mean-field theory, Phys. Today 57, 53 (2004).

[34] G. Kotliar, S. Y. Savrasov, K. Haule, V. S. Oudovenko, O. Parcollet, and C. A. Marianetti, Electronic structure calculations with dynamical mean-field theory, Rev. Mod. Phys. 78, 865 (2006).

[35] M. S. Golden, C. Dür, A. Koitzsch, S. Legner, Z. Hu, S. Borisenko, M. Knupfer, and J. Fink, The electronic structure of cuprates from high energy spectroscopy, J. Electron Spectrosc. Relat. Phenom. 117-118, 203 (2001).

[36] I. H. Inoue, I. Hase, Y. Aiura, A. Fujimori, Y. Haruyama, T. Maruyama, and Y. Nishihara, Systematic Development of the Spectral Function in the $3 d^{1}$ Mott-Hubbard System $\mathrm{Ca}_{1-x} \mathrm{Sr}_{x} \mathrm{VO}_{3}$, Phys. Rev. Lett. 74, 2539 (1995).

[37] M. Takizawa, M. Minohara, H. Kumigashira, D. Toyota, M. Oshima, H. Wadati, T. Yoshida, A. Fujimori, M. Lippmaa, M. Kawasaki, H. Koinuma, G. Sordi, and M. Rozenberg, Coherent and incoherent $d$ band dispersions in $\mathrm{SrVO}_{3}$, Phys. Rev. B 80, 235104 (2009).

[38] S. Aizaki, T. Yoshida, K. Yoshimatsu, M. Takizawa, M. Minohara, S. Ideta, A. Fujimori, K. Gupta, P. Mahadevan, K. Horiba, H. Kumigashira, and M. Oshima, Self-Energy on the Low- to High-Energy Electronic Structure of Correlated Metal $\mathrm{SrVO}_{3}$, Phys. Rev. Lett. 109, 056401 (2012).

[39] S. Backes, T. C. Rödel, F. Fortuna, E. Frantzeskakis, P. Le Fèvre, F. Bertran, M. Kobayashi, R. Yukawa, T. Mitsuhashi, M. Kitamura, K. Horiba, H. Kumigashira, R. Saint-Martin, A. Fouchet, B. Berini, Y. Dumont, A. J. Kim, F. Lechermann, H. O. Jeschke, M. J. Rozenberg, R. Valentí, and A. F. SantanderSyro, Hubbard band versus oxygen vacancy states in the correlated electron metal $\mathrm{SrVO}_{3}$, Phys. Rev. B 94, 241110 (2016).
[40] P. Blaha, K. Schwarz, G. K. H. Madsen, D. Kvasnicka, and J. Luit, Wien2k: An Augmented Plane Wave+Local Orbitals Program for Calculating Crystal Properties (Karlheinz Schwarz, Technische Universität Wien, Austria, 2001).

[41] R. Khasanov, M. Bendele, K. Conder, H. Keller, E. Pomjakushina, and V. Pomjakushin, Iron isotope effect on the superconducting transition temperature and the crystal structure of $\mathrm{FeSe}_{1-x}$, New J. Phys. 12, 073024 (2010).

[42] See Supplemental Material at http://link.aps.org/supplemental/ 10.1103/PhysRevB.95.081106 for further experimental data and details of DMFT calculations performed with different interaction parameters.

[43] J. Ferber, K. Foyevtsova, H. O. Jeschke, and R. Valentí, Unveiling the microscopic nature of correlated organic conductors: The case of $\kappa-(\mathrm{ET})_{2} \mathrm{Cu}\left[\mathrm{N}(\mathrm{CN})_{2}\right] \mathrm{Br}_{x} \mathrm{Cl}_{1-x}$, Phys. Rev. B 89, 205106 (2014).

[44] E. Gull, A. J. Millis, A. I. Lichtenstein, A. N. Rubtsov, M. Troyer, and P. Werner, Continuous-time Monte Carlo methods for quantum impurity models, Rev. Mod. Phys. 83, 349 (2011).

[45] B. Bauer, L. D. Carr, H. G. Evertz, A. Feiguin, J. Freire, S. Fuchs, L. Gamper, J. Gukelberger, E. Gull, S. Guertler, A. Hehn, R. Igarashi, S. V. Isakov, D. Koop, P. N. Ma, P. Mates, H. Matsuo, O. Parcollet, G. Pawłowski, J. D. Picon, L. Pollet, E. Santos, V. W. Scarola, U. Schollwöck, C. Silva, B. Surer, S. Todo, S. Trebst, M. Troyer, M. L. Wall, P. Werner, and S. Wessel, The ALPS project release 2.0: open source software for strongly correlated systems, J. Stat. Mech. (2011) P05001.

[46] T. Miyake, K. Nakamura, R. Arita, and M. Imada, Comparison of $a b$ initio low-energy models for $\mathrm{LaFePO}, \mathrm{LaFeAsO}, \mathrm{BaFe}_{2} \mathrm{As}_{2}$, $\mathrm{LiFeAs}$, FeSe, and FeTe: Electron correlation and covalency, J. Phys. Soc. Jpn. 79, 044705 (2010).

[47] V. I. Anisimov, I. V. Solovyev, M. A. Korotin, M. T. Czyżyk, and G. A. Sawatzky, Density-functional theory and NiO photoemission spectra, Phys. Rev. B 48, 16929 (1993).

[48] S. L. Dudarev, G. A. Botton, S. Y. Savrasov, C. J. Humphreys, and A. P. Sutton, Electron-energy-loss spectra and the structural stability of nickel oxide: An LSDA $+U$ study, Phys. Rev. B 57, 1505 (1998).

[49] K. S. D. Beach, Identifying the maximum entropy method as a special limit of stochastic analytic continuation, arXiv:condmat/0403055.

[50] A. Carrington, Quantum oscillation studies of the Fermi surface of iron-pnictide superconductors, Rep. Prog. Phys. 74, 124507 (2011).

[51] S. V. Borisenko, D. V. Evtushinsky, Z.-H. Liu, I. Morozov, R. Kappenberger, S. Wurmehl, B. Buchner, A. N. Yaresko, T. K. Kim, M. Hoesch, T. Wolf, and N. D. Zhigadlo, Direct observation of spin-orbit coupling in iron-based superconductors, Nat. Phys. 12, 311 (2016).

[52] We note that experimental measurements have an additional background which will not be accounted for in the calculation, which also distorts the color scales.

[53] M. Casula, Ph. Werner, L. Vaugier, F. Aryasetiawan, T. Miyake, A. J. Millis, and S. Biermann, Low-Energy Models for Correlated Materials: Bandwidth Renormalization from Coulombic Screening, Phys. Rev. Lett. 109, 126408 (2012).

[54] S. Biermann, Dynamical screening effects in correlated electron materials - a progress report on combined many-body perturbation and dynamical mean field theory: "GW+DMFT," J. Phys. Condens. Matter 26, 173202 (2014). 
[55] A. van Roekeghem, L. Vaugier, H. Jiang, and S. Biermann, Hubbard interactions in iron-based pnictides and chalcogenides: Slater parametrization, screening channels, and frequency dependence, Phys. Rev. B 94, 125147 (2016).

[56] K. Jiang, J. Hu, H. Ding, and Z. Wang, Interatomic Coulomb interaction and electron nematic bond order in FeSe, Phys. Rev. B 93, 115138 (2016).

[57] L. Ortenzi, E. Cappelluti, L. Benfatto, and L. Pietronero, Fermi-Surface Shrinking and Interband Coupling in Iron-Based Pnictides, Phys. Rev. Lett. 103, 046404 (2009).
[58] J. K. Glasbrenner, I. I. Mazin, H. O. Jeschke, P. J. Hirschfeld, R. M. Fernandes, and R. Valentí, Effect of magnetic frustration on nematicity and superconductivity in iron chalcogenides, Nat. Phys. 11, 953 (2015).

[59] A. Yamasaki, Y. Matsui, S. Imada, K. Takase, H. Azuma, T. Muro, Y. Kato, A. Higashiya, A. Sekiyama, S. Suga, M. Yabashi, K. Tamasaku, T. Ishikawa, K. Terashima, H. Kobori, A. Sugimura, N. Umeyama, H. Sato, Y. Hara, N. Miyagawa, and S. I. Ikeda, Electron correlation in the FeSe superconductor studied by bulk-sensitive photoemission spectroscopy, Phys. Rev. B 82, 184511 (2010). 